\title{
Design of Defected Ground Low pass Microstrip Filters with Enhanced Performance Characteristics
}

\author{
Dr. Dheeraj Bhardwaj \\ Assistant Professor, Department of Physics, \\ BIT-Mesra- Jaipur Campus, Jaipur, India. \\ Srishti Saraswat \\ Bachelor of Engineering-ECE, Department of Electronics \& Communication Engineering, \\ BIT-Mesra- Jaipur Campus, Jaipur, India \\ Gitansh Gulati \\ Project Engineer, Naltech \\ National Aerospace Laboratories, Bangalore, India. \\ Komal Sharma \\ Reader, Department of Physics, \\ Swami Keshvanand Institute of Technology, Jaipur , India
}

\begin{abstract}
Advancements in the field of RF/Microwave have triggered the designers to improve each and every component involved in microwave modern communication systems such as Low Noise Amplifier, Multiplier, Mixer, microwave filters, IF filters, antennas, power amplifier etc. In this paper we propose the design of microstrip lowpass filter using stepped-impedance (L-C ladder type) technique with the introduction of basic concept of defected ground structure (DGS) to achieve reduced size circuits. The designed prototype filter exhibits passband from frequency ranges, 0.1GHz-1.76 GHz which has been fabricated on $24.80 \mathrm{~mm}$ (L) X 16mm (W) FR4 substrate with $1.6 \mathrm{~m}$ thickness The introduction of semi circular dumbbell shaped defected ground structure cell offers significant improvement of stopband attenuation and size reduction as can be seen. This lowpass filter can be used in wide variety of modern communication applications such as mobile broadcasting VHF band(174 MHz-240 MHz) and L band(1452 MHz-1492 MHz)GPS L1 band(1575 MHz) and L2 band(1227.6 MHz).
\end{abstract}

Keywords - DEFECTED GROUND STRUCTURE (DGS), STEPPED IMPEDANCE, LC LADDER, LOW PASS FILTER

\section{INTRODUCTION}

In the last few years, enormous development in wireless communications systems has created the need for new microwave components with stringent specifications. Technological developments have created more demanding requirements that impose new challenges on design, optimization and realization of these components. In case of microwave filters, more challenging parameters such as selectivity, suppression (out-of band), bandwidth, phase linearity and compactness have been researched upon. In this paper, the concept of defected ground structure to enhance the performance characteristics have been taken into account by the authors.

Artificial periodic structures exhibiting an unusual property of preventing EM waves from propagating over a certain range of frequencies and allowing EM waves to propagate through them over different range of frequencies have been termed as electromagnetic band gap structures (EBG). For a printed microwave circuit and transmission 
line, another degree of freedom is offered by implementing EBG structures on the ground plane which depending on shape and dimensions can control the excitation and propagation of electromagnetic waves through the substrate layer. The work reported in this paper mainly focuses on improving the insertion loss, return loss and accomplishing sharp roll of characteristics with higher out of band rejection.

\section{STEPPED IMPEDANCE L-C LADDER TYPE LOW PASS FILTER DESIGN}

This configuration uses a cascaded structure of alternating high and low impedance transmission lines. Due to their size much shorter than the associated guided-wavelength, they are known as semi-lumped elements. The higher impedance lines act as series inductor and low impedance lines act as shunt capacitors as shown in figure 1.
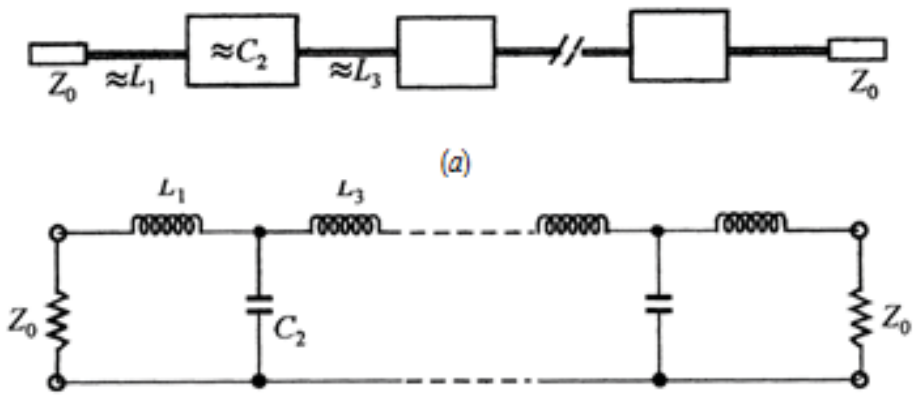

(b)

Figure 1. (a) General structure of the stepped-impedance lowpassmicrostrip filters. (b) L-C ladder type of lowpass filters to be approximated.

In this section a microstrip lowpass filter has been designed based on the specification listed in Table 1 and design equations. Further, same filter design has been carried out using defected ground structure.

Table - 1 Design considerations

\begin{tabular}{|c|c|}
\hline Parameters & Values \\
\hline Cutoff Frequency $\left(\mathbf{f}_{\mathrm{c}}\right.$ & $\mathbf{2 ~ G H z}$ \\
\hline Pass Band Ripple & $\mathbf{0 . 1} \mathrm{dB}$ \\
\hline Source/Load Impedance & $\mathbf{5 0 ~ o h m ~}$ \\
\hline Type/Response & Three Pole Chebyshev Prototype \\
\hline
\end{tabular}

Using the chebyshev lowpass prototype filter values as referenced in (Pozar) and element transformations, we have;

$$
\begin{aligned}
& \mathrm{L}_{1}=\mathrm{L}_{3}=\left(Z_{\alpha} / g_{0}\right)\left(\Omega_{\Omega} / 2 \pi_{\infty}\right)_{1} \Omega_{1}=4.106 \times 10^{-9} \mathrm{H}
\end{aligned}
$$

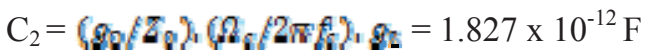

The filter is to be fabricated on a substrate with a relative dielectric constant of 4.3(FR-4 Lossy) and a thickness of $1.5 \mathrm{~mm}$. Following the above mentioned considerations, the characteristic impedances of the high- and lowimpedance lines are chosen as $Z_{\mathrm{oL}}=100 \Omega$ and $Z_{\mathrm{oc}}=24 \Omega$. The relevant design parameters for the microstrip lines are calculated using standard formulaes and depicted in Table 2. 
Table - 2 Design parameters for microstrip lines for stepped impedance lowwpass filter.

\begin{tabular}{|c|c|c|}
\hline Characteristic Impedance $(\mathbf{\Omega})$ & Guided Wavelength (mm) & Microstrip Width (mm) \\
\hline$z_{a c}=24$ & $\lambda_{9}=78.02$ & $W_{a}=8.876$ \\
\hline$z_{0}=80$ & 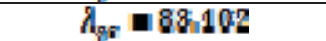 & $\mathrm{W}_{\mathrm{b}}=\mathrm{2} .893$ \\
\hline$z_{0}=100$ & $\lambda_{06}=8698$ & $W_{L}=0.659$ \\
\hline
\end{tabular}

Physical lengths of high and low impedance lines can be calculated as follows:-

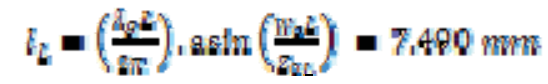

$$
\begin{aligned}
& \varepsilon_{c}=\left(\frac{A_{q}}{2 \pi}\right) \cdot \operatorname{asln}\left(w_{c} c z_{\sigma c}\right)=7.349 \mathrm{~mm}
\end{aligned}
$$

The results of $(3,4)$ do not take into account series reactance of the low-impedance line and shunt susceptance of the high-impedance lines. To include these effects, the lengths of the high- and low-impedance lines should be adjusted

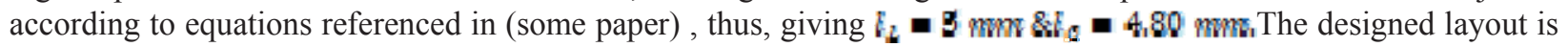
illustrated in Figure 2 and the it's performance is carried out using full-wave analysis simulation as depicted in Figure 3.

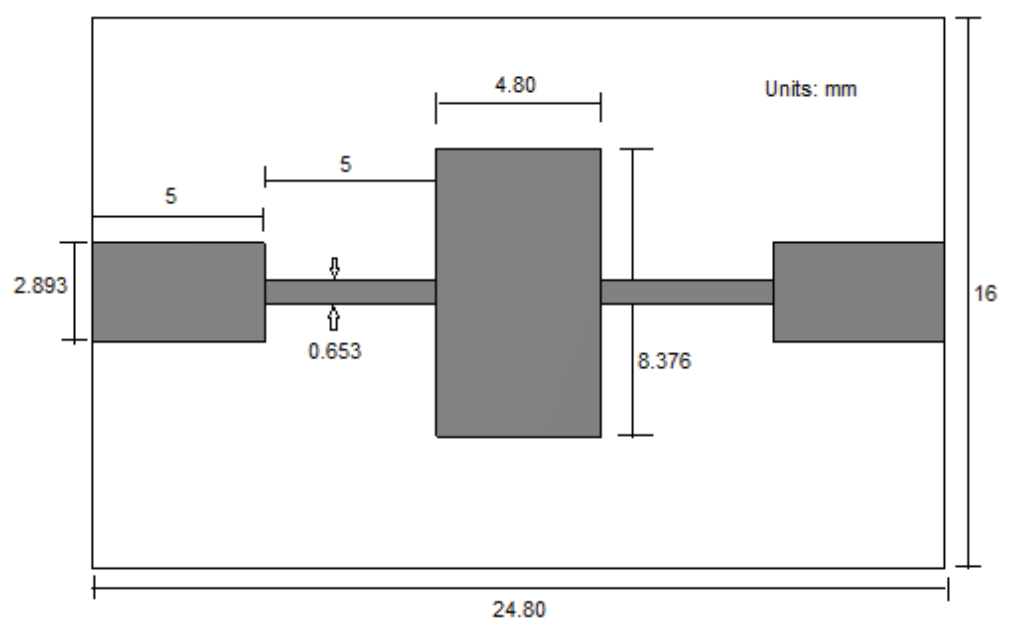

Figure 2. Front view Layout of a three-pole, stepped-Impedance microstrip lowpass filter on FR-4 Lossy substrate with height $=1.5 \mathrm{~mm}$ 


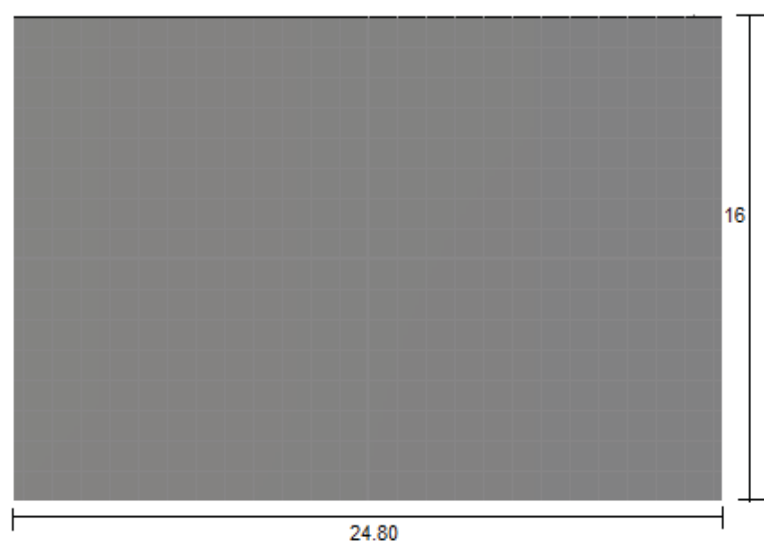

Figure 2. Back view Layout of a three-pole, stepped-Impedance microstriplowpass filter on FR-4 Lossy substrate with height=1.5mm

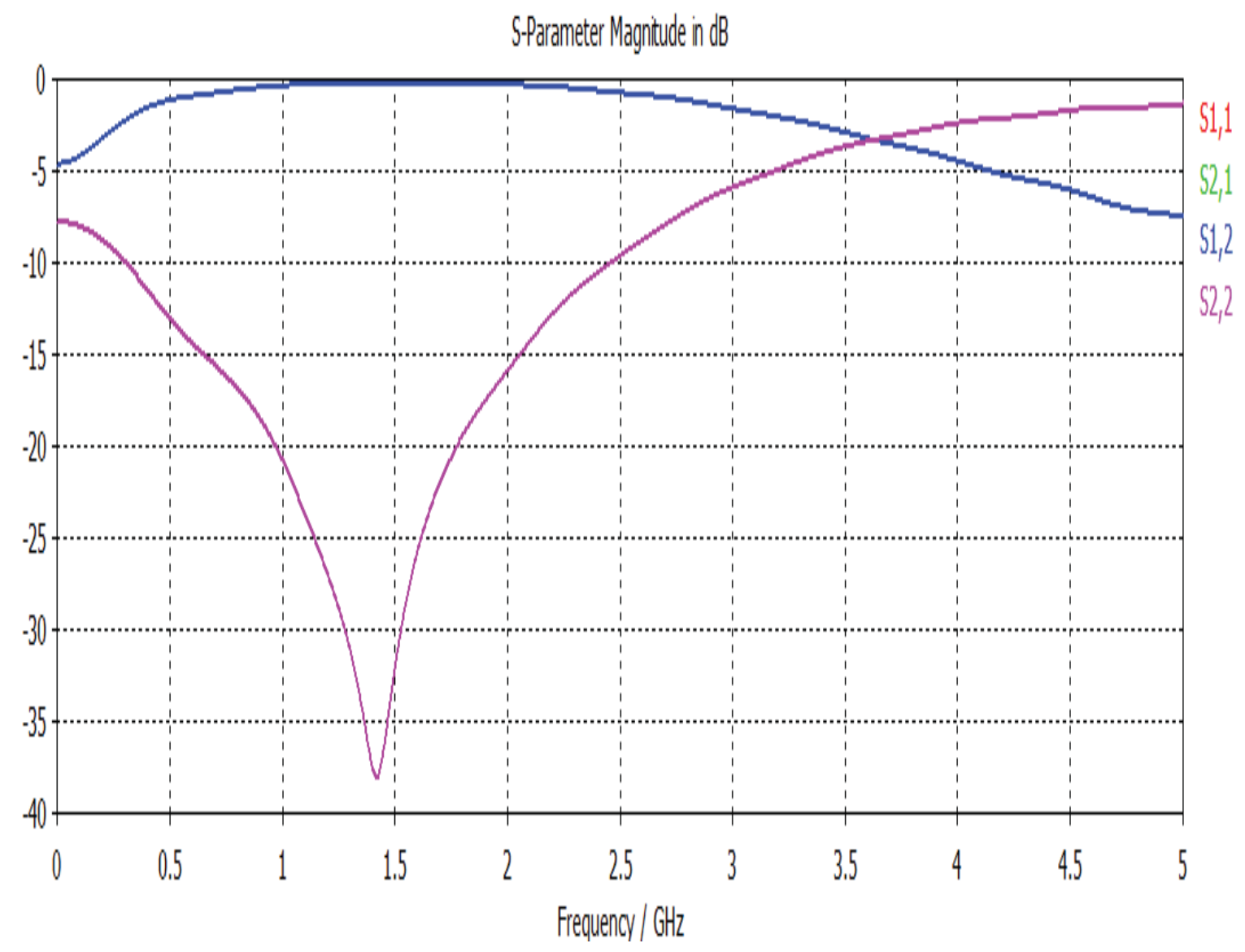

Figure 3. Full-wave EM Simulated performance characteristics. 


\section{STEPPED IMPEDANCE L-C LADDER TYPE LOW PASS FILTER DESIGN WITH DEFECTED GROUND STRUCTURE}

In this paper a new compact filter design is proposed with better pass band characteristics along with improved stop band rejection as well as high attenuation in the stop band. The proposed compact filter design constitutes a semi circular dumbbell shaped unit cell structure in the ground plane. A DGS unit cell structure can be characterized by an LC equivalent circuit.

The semi circular dumbbell shaped DGS is inserted along the centre of the ground plane. A resonating frequency is achieved by a combination of inductance and capacitance to attain the perfect impedance match at that particular frequency. Embedding the structure in the ground has shifted the cutoff frequency towards the higher side due to decrease in the microstrip inductance and characteristic impedance. The effect of the gap is depicted by the shift in the attenuation pole to higher frequency side.

The semicircular dumbbell shaped structure has improved the series effective inductance and effective capacitance .The dumbbell shaped structure has suppressed the higher order harmonics of $2 \mathrm{GHz}$ and provide an improved maximally flat lowpass response.
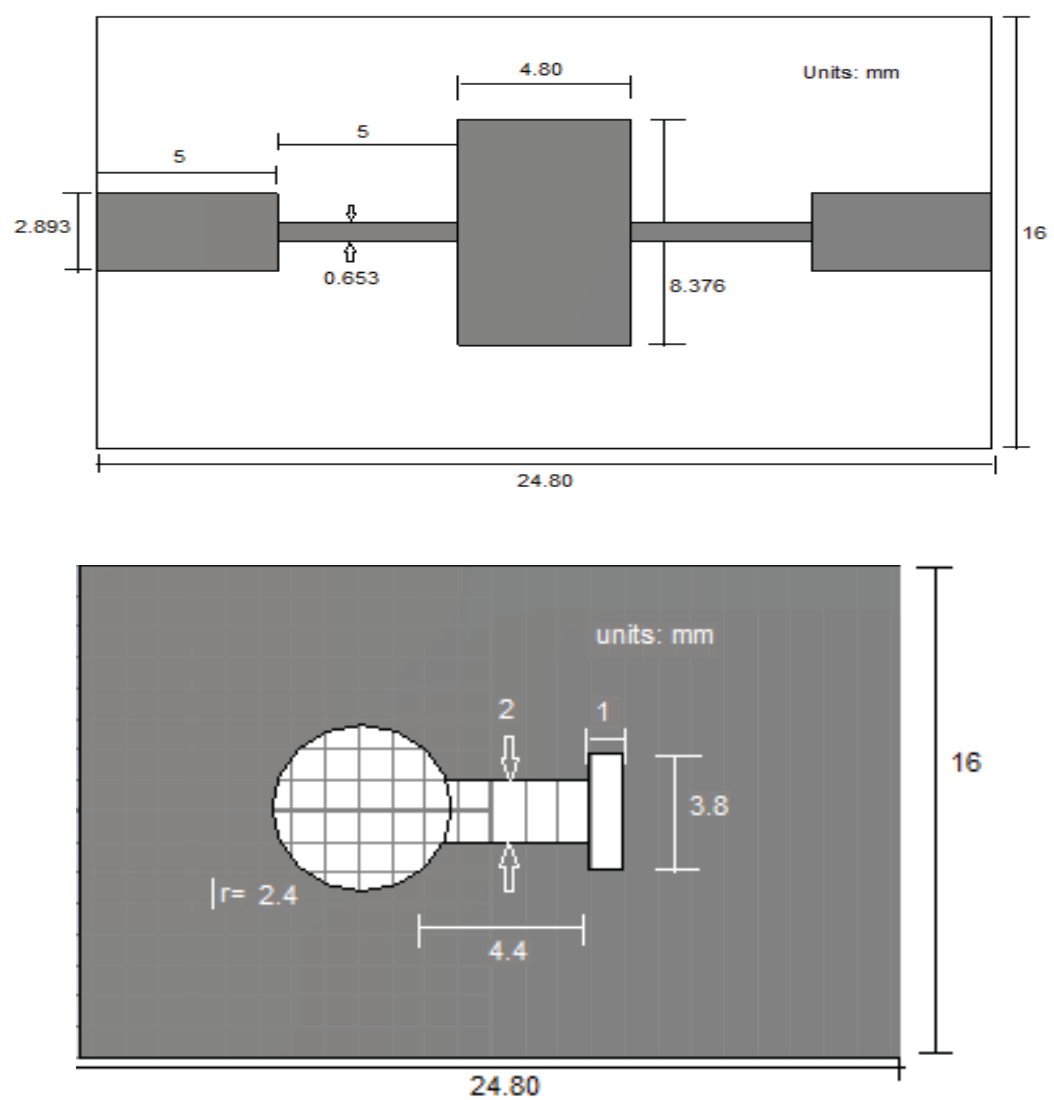

Figure 4. Layout of a three-pole, stepped-Impedance microstrip low pass filter with semi-circular dumbbell shaped DGS on FR-4 Lossy with height=1.6mm (a) Front view (b) Back view 


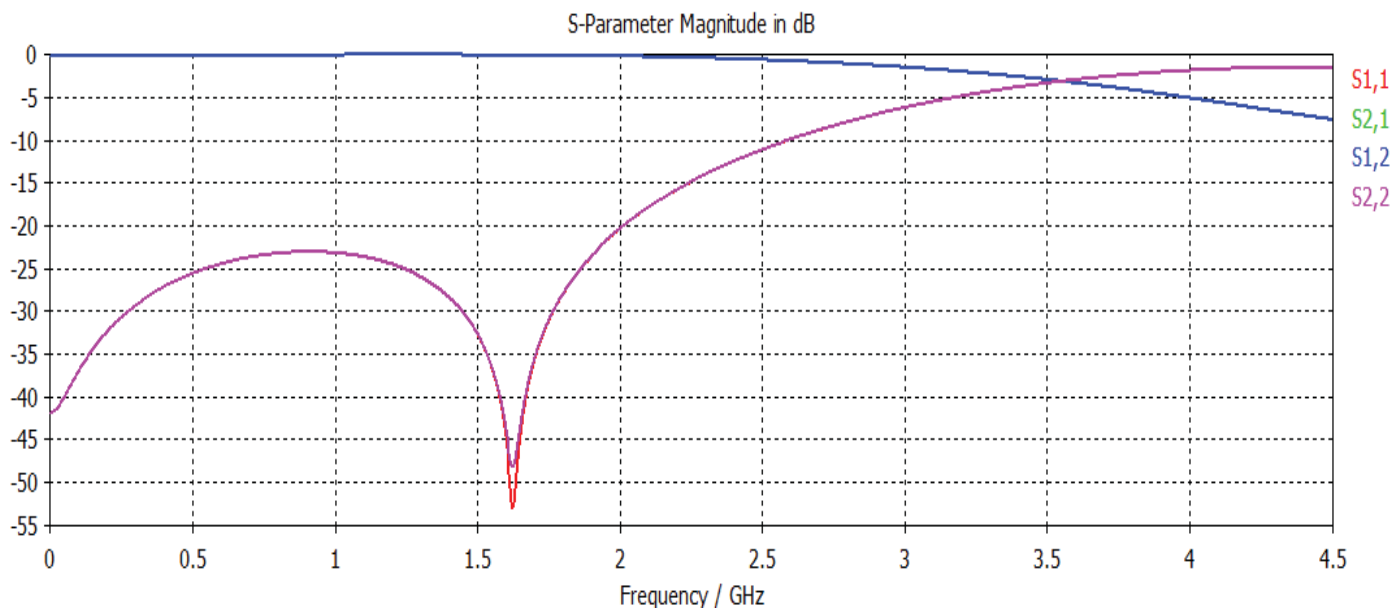

Figure 5. Fullwave EM Simulated performance characteristics.

\section{IV.CONCLUSION}

The conventional semi lumped low pass filter design exhibits a pass band from $0-1.76 \mathrm{GHz}$ on a substrate of dielectric constant 4.4 and height $1.5 \mathrm{~mm}$. The introduction of defected ground structure to the conventional LC ladder type low pass filter has altered the ground plane current distribution further leading to change in the inductance and capacitance which in turn alter the filter characteristics . On introduction of a semi circular dumbbell shaped unit cell slot on the ground plane effective improvement of stop band attenuation has been achieved and the pass band $0-2.1 \mathrm{GHz}$ is maximally flat.

The basic problem lies in the accurate circuit modeling of the filter with DGS which can be overcome to a great extend by considering each and every discontinuity encountered in the design to have been formulated separately(such as in Quasi-Static modeling) but the various losses introduced are not taken into account. Moreover, this design realization gives you the most well-defined desired outcome but some modifications are necessary to optimize the design to improve the skirt selectivity, insertion losses and stopband attenuation characteristics.

\section{REFERENCES}

[1] Jia-Sheng Hong and M.J.Lancaster, Microstrip filters for RF/Microwave Applications, John Wiley \& Sons,Inc. ,2001.

[2] Debatosh Guha and Yahia M M Antar, Microstrip and Printed Antennas: New Trends, Techniques and Applications, John Wiley \& Sons Ltd. ,2011.

[3] David M. Pozar, Microwave Engineering, 4th Edition, John Wiley \& Sons Inc. ,2012.

[4] C.A.Balanis,Antenna Theory: Analysis and Design, 2nd Edition, John Wiley \& Sons Inc. ,2007.

[5] Xun Luo, Jian-Guo Ma and Er-Ping Li, Hybrid Microstrip/DGS Cell For Filter Design IEEE Microwave and Wireless Components Letters, Vol.21, No.10,October 2011

[6] Dal Ahn,Jun-Seok Park and Tatsuo Itoh, A Design of the Low-Pass Filter Using the Novel Microstrip Defected Ground Structure IEEE Transactions on Microwave theory and Techniques, Vol. 49, No.1, January 2001.

[7] Pragya Singn, Professor Raghuvir Tomar and Dr. Prakash Bhartia, The Use of Defected Ground Structures in Deigning Microstrip Filters with Enhanced Performance Characteristics International Journal of Advanced Research in Computer and Communication Engineering, Vol. 2, Issue 10, October 2013.

[8] A. Nouri and G. R. Dadashzadeh, A Compact UWB Band-Notched Printed Monopole Antenna With Defected Ground Structure, IEEE Antennas and Wireless Propagation Letters, vol. 10, 2011.

[9] G. Mansour, M. J. Lancaster, P. S. Hall, P. Gardner, and E. Nugoolcharoenlap, Design of filtering microstrip antenna using filter synthesis approach, Progress In Electromagnetics Research, Vol. 145, 59-67, 2014. 